\title{
Comparison Between Linear and Nonlinear Models of Mixed Pixels in Remote Sensing Satellite Images Based on Cierniewski Surface BRDF Model by Means of Monte Carlo Ray Tracing Simulation
}

\author{
Kohei Arai \\ Graduate School of Science and Engineering \\ Saga University \\ Saga City, Japan
}

\begin{abstract}
Comparative study on linear and nonlinear mixed pixel models of which pixels in remote sensing satellite images is composed with plural ground cover materials mixed together, is conducted for remote sensing satellite image analysis. The mixed pixel models are based on Cierniewski of ground surface reflectance model. The comparative study is conducted by using of Monte Carlo Ray Tracing: MCRT simulations. Through simulation study, the difference between linear and nonlinear mixed pixel models is clarified. Also it is found that the simulation model is validated.
\end{abstract}

Keywords-Monte Carlo simulation; ray tracing method; mixed pixel model; surface model;

\section{INTRODUCTION}

All land pixels in remote sensing images are essentially mixed pixels that consist of multiple ground cover materials. Currently, there are two types of models aiming to untangle these contributions: linear and non-linear mixture models. The linear mixture models assume negligible interactions among distinct ground cover materials while the nonlinear mixture models assume that incident solar radiation is scattered within the scene itself and that these interaction events may involve several types of ground cover materials.

R. Singer and T. B. McCord (1979) [1], B. Hapke (1981) [2] and R. N. Clark and T. I. Roush (1984) [3] proposed linear mixture models while R. Singer (1974) [4], B. Nash and J. Conel (1974) [5] proposed nonlinear mixture models for the mixed pixels containing different mineral resources. Meanwhile, C. C. Borel and S. A. Gerst (1994) [6] proposed another nonlinear mixture model for vegetated areas. These nonlinear mixture pixel models, however, did not take into consideration the influence of topographic features or the influence of multiple scattering in the atmosphere. Meanwhile, Cierniewski proposed a surface model which consists of two dimensional array of ellipsoidal shapes of elements [7]. One of the specific features of the Cierniewski's surface model is representation of Bi-Directional Reflectance Function: BRDF. Although there are some nonlinear mixture models, there is no nonlinear mixed pixel model taking into account BRDF of the surface in concern.
A nonlinear mixture model for the interpretation of mixed pixels in remote sensing satellite images is proposed. The proposed model is a Monte Carlo Ray-Tracing: MCRT model that takes into account interactions among the ground cover materials (multiple reflections among the materials on the surface) [8]. The proposed model also takes into account topographic features (slope) of the ground surface. As an example, Top of the Atmosphere: TOA radiance of mixed pixels based on the proposed nonlinear model is compared to that of the conventional linear model.

This paper proposes a nonlinear mixed pixel model that takes into account topographic features of the surface and multiple scattering in the atmosphere. Furthermore, the proposed nonlinear mixed pixel model takes into account interactions among ground cover materials separated by different distances and having different shapes. Since multiple scattering interactions in three dimensional media are not so easy to solve using the radiative transfer equation, the proposed mixture model is based on Monte Carlo RayTracing: MCRT.

Section 2 describes the proposed nonlinear mixed pixel model together with details of the MCRT algorithm. Section 3 presents experimental results showing the influence of multiple scattering interactions between trees, the shape of the trees, the slope of the terrain, and the atmospheric optical depth. Finally, the model derived TOA radiance is compared between linear and nonlinear models.

\section{PROPOSED MODEL}

\section{A. Monte Carlo Ray-Tracing:MCRT Simulation Model}

Nonlinear mixture model and brief description of Monte Carlo Ray-Tracing model: MCRT. Nonlinear mixing model proposed here is composed with more than two ground cover materials and is based on the MCRT model. In order to take into account the geographical feature, slope of the ground surface can be changed. Also any ground cover materials can be set for the ground surface together with different shape of ground cover materials. The simulation with MCRT model is called MCRT Simulation, MCRTS (Arai, 2005) [9]. In MCRTS, 50 by 50 by $50 \mathrm{~km}$ of simulation cell size is assumed. 
The ground surface is composed with two planes, surface A and $\mathrm{B}$, with the different slopes, $\alpha$ and $\beta$ and with surface reflectance, $\Gamma_{\mathrm{A}}$ and $\Gamma_{\mathrm{B}}$ as is shown in Figure 1. a and b show IFOV on the ground for the surface A and B.

\section{TOA.Rad.}

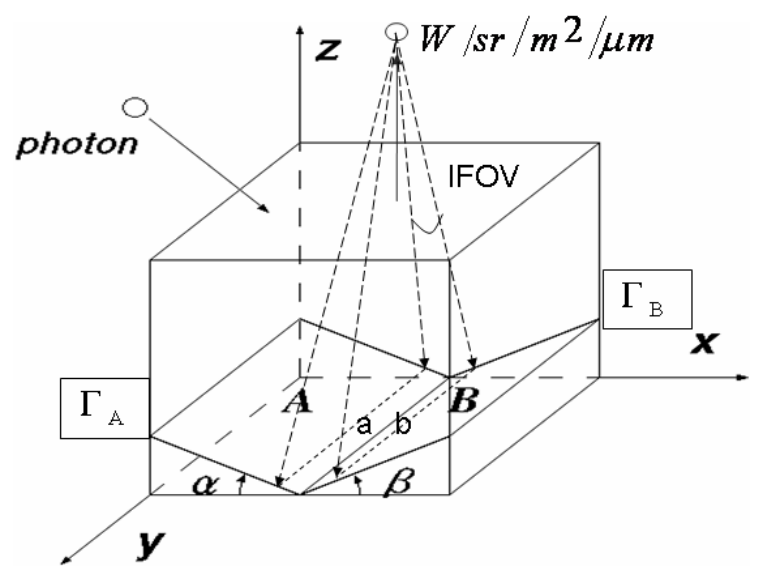

Fig.1. Nonlinear mixed pixel model based on Monte Carlo Ray-Tracing Simulation model with 50x50x50km cell and two ground surfaces (The pixels situated along with border between two surface are mixed pixels).

A photon is put in the simulation cell from the top of the cell with the incidence angle that depends on the specified solar zenith angle. The position of which the photon is put in is changed by time by time in accordance with the uniformly distributed random numbers which are generated by Mersenne Twister ${ }^{1}$.

Depending on the optical depth of the atmosphere, free travel length $L$ of photon is determined as follows,

$$
\begin{aligned}
& L=-L_{0} \log (\text { Rnd }) \\
& L_{0}=\frac{h}{\tau_{\text {all }}}
\end{aligned}
$$

where ${ }^{L_{0}}$ is called free travel length, denoting the average distance of interaction of a photon from one position to another. Rnd is uniformly distributed random numbers ranges from 0 to 1 . $h$ denotes the physical height of the atmosphere $\left(50 \mathrm{~km}\right.$ in this case) while $\tau_{\text {all }}$ denotes the optical depth of the atmosphere which is determined as follows,

$$
\tau_{\text {all }}=\tau_{\text {aero }}+\tau_{\text {mol }}
$$

where the subscript aero is associated with aerosols while mol with molecules. Here, it is assumed that atmosphere consists of aerosols and air molecules. Because the wavelength in concern ranges from 450 to $1050 \mathrm{~nm}$ so that optical depth of ozone and water vapors are assumed to be

\footnotetext{
${ }^{1}$ Mersenne Twister (MT), http://www.math.sci.hiroshima-u.ac.jp/ m$\mathrm{mat} / \mathrm{MT} / \mathrm{mt} . \mathrm{html}$
}

negligible except $936 \mathrm{~nm}$ of water vapor absorption band. A small absorption due to ozone is situated from 500 to $650 \mathrm{~nm}$ around.

The photon meets aerosol particles or molecule when the photon travels in the atmosphere then scattering due to the aerosols or molecules occurs. The probability of the collision to the aerosols or molecules depends on their optical depths. If the endpoint of photon travel is in the atmosphere, the photon meets aerosol or molecule. The probability of the photon meets aerosol is $\tau_{\text {aero }} / \tau_{\text {all }}$ while that of the photon meets molecule is $\tau_{\text {mol }} / \tau_{\text {all }}$. In accordance with the phase function of aerosols or molecules, the photon is scattered. Strength of scattering as a function of scattering angle $\theta$ is determined by the phase function, $P(\theta)$, the Rayleigh for molecules, equation (4) and Heyney-Greestein function, equation (5) (it is just an approximation function of which the phase function is monotonically decreasing) for aerosols. Actual phase function can be determined with MODTRAN 4.0 of Mie code with the measured refractive index of aerosols through field experiments. By using uniformly distributed random numbers, scattering direction is determined. The phase function as $P(\theta)$, where $\theta$ is the angle between the incident direction and the scattering direction.

For molecules, the Rayleigh phase function is as follows,

$$
P(\theta)=(3 / 4)\left(1+\cos ^{2} \theta\right)
$$

while that for aerosols, we use the Heyney-Greenstein approximation function of the following,

$$
P(\theta)=\frac{1-g_{\lambda}^{2}}{\left(1+g_{\lambda}^{2}-2 g_{\lambda} \cos \theta\right)^{3 / 2}}
$$

where $g_{\lambda}$ is the asymmetry factor of the aerosol phase function which depends on the wavelength of the radiation and the compositions, sizes, and the shapes of the aerosol particles.

In the calculation of TOA radiance, the number of photons, $N$ which comes out from the top of the atmosphere within the angle range which corresponds to the Instantaneous Field of View: IFOV of the sensor in concern is used thus the normalized TOA radiance, $\mathrm{Rad}$ is determined as follows,

$$
R a d=\frac{\mu_{0} \mu \Delta \mu}{2}\left(\frac{N}{N_{\text {total }}}\right)
$$

where $\mu_{0}=\cos \theta_{0}, \mu=\cos \theta, \theta_{0}$ is the solar zenith angle and $\theta$ is a viewing solid angle. $\Delta \mu$ is a view solid angle, i.e., FOV (field of view). $N_{\text {total }}$ is the number of photons which are put in the cell in total. If you multiply solar irradiance to $\mathrm{Rad}$ in unit of $\left(\mathrm{W} / \mathrm{m}^{2} / \mathrm{str} / \mu \mathrm{m}\right)$, then the TOA radiance in the same unit is calculated.

The input parameters are determined by field experimental data. They are (1) Material reflectance which is albedo of the entire ground cover material, (2) Background surface material 
reflectance, (3) Material-material distance, (4) Optical depth of aerosol and molecule, (5) Solar zenith and azimuth angles, (6) IFOV of the sensor, (7) Sensor direction (view zenith angle) and height. On the other hand, output parameters include TOA radiance and ten groups of photons. They are (1) Photons that are put in the atmosphere from the top of cell in total, (2) Photons that are come from the top of the cell within the range of IFOV, (3) Photons that are reflected by material, (4) Photons that are absorbed by material, (5) Photons that are reflected on the background, (6) Photons that are absorbed on the background, (7) Photons that are scattered by aerosols, (8) Photons that are absorbed by aerosols, (9) Photons that are scattered by molecules and (10) Photon that are absorbed by molecule. A photon equation must be formed, that is, the number of put-in-photons must be equal to the sum of comeout-photons which are come-out from the top of cell and the photons which are absorbed by aerosols and molecules, material and background. Each simulation has proved this equation.

From the results from the preliminary MCRTS with a plenty of input parameters, it is concluded that 700,000 of putin-photons would be enough for the MCRTS in many cases.

\section{B. Surface Model}

Figure 2 shows Cierniewski surface Bi-Directional Reflectance Distribution Funct6ion: BRDF model based ground surface model.

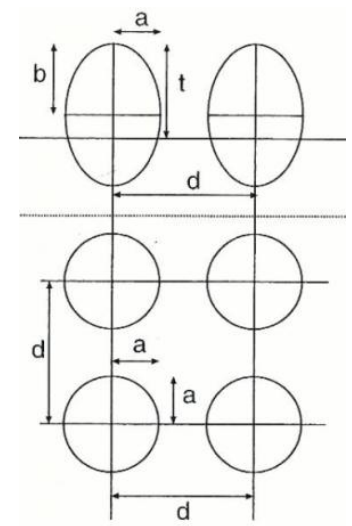

Fig.2. Cierniewski based ground surface model (Side view: above, Top view: bottom)

The proposed model is composed with two dimensional array of ellipsoidal shape of elements. These elements are shaped with ellipsoidal shape (shorter radius of "a" and longer radius of "b") and are two dimensionally aligned with the interval of "d". These surfaces are situated on the ground surface in the defined space of MCRT model. The sun, sensor onboard remote sensing satellites are situated as shown in Figure 3.

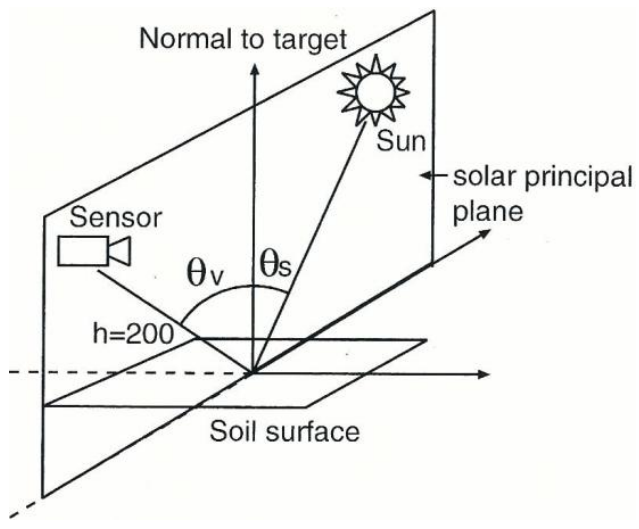

Fig.3. Geometric relations among the sun, sensor, and ground surface (soil surface)

In the principal plane among the sun, sensor, and the point of ground cover target on ground surface, solar zenith angle, observation zenith angle, slant range, and Instantaneous Field of View: IFOV are defined as shown in Figure 4.

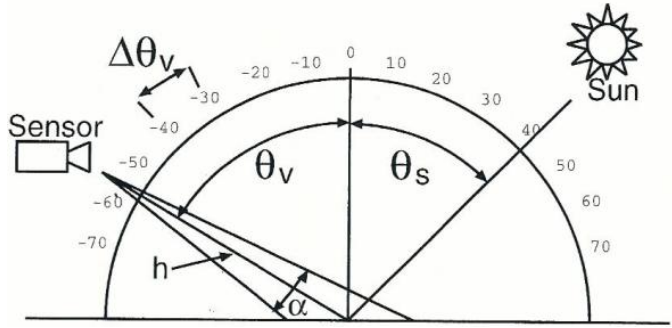

Fig.4. Definitions of solar zenith angle, observation zenith angle, slant range, and Instantaneous Field of View: IFOV

These surface models are situated left and right ground surface with the designated slopes separately for linear model while these surfaces are situated with the same slopes interactively for nonlinear model as shown in Figure 5. Geometric relation for these mixed pixel models are shown in Figure 6.

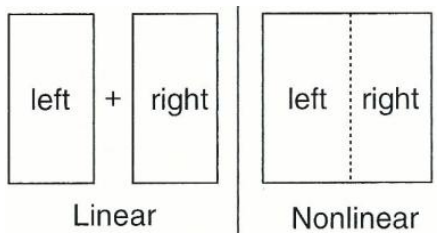

Fig.5. Linear and Nonlinear mixed pixel models

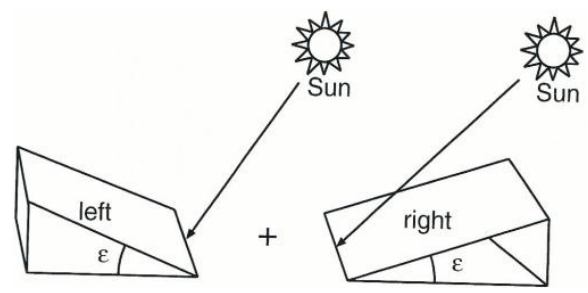

(a)Linear model 


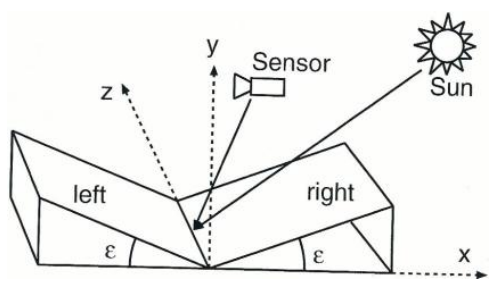

(b)Nonlinear model

Fig.6. Linear and nonlinear mixed pixel models

\section{EXPERIEMNTS}

\section{A. Comparison to Cierniewski Surface Model}

Slope zero of flat surface is assumed to be simulated. Table 1 shows the parameters for the simulation. All the simulation studies hereafter are conducted at the wavelength of $500 \mathrm{~nm}$. Figure 7 shows an example of the Bi-Directional Reflectance Function: BRDF with slope zero surface.

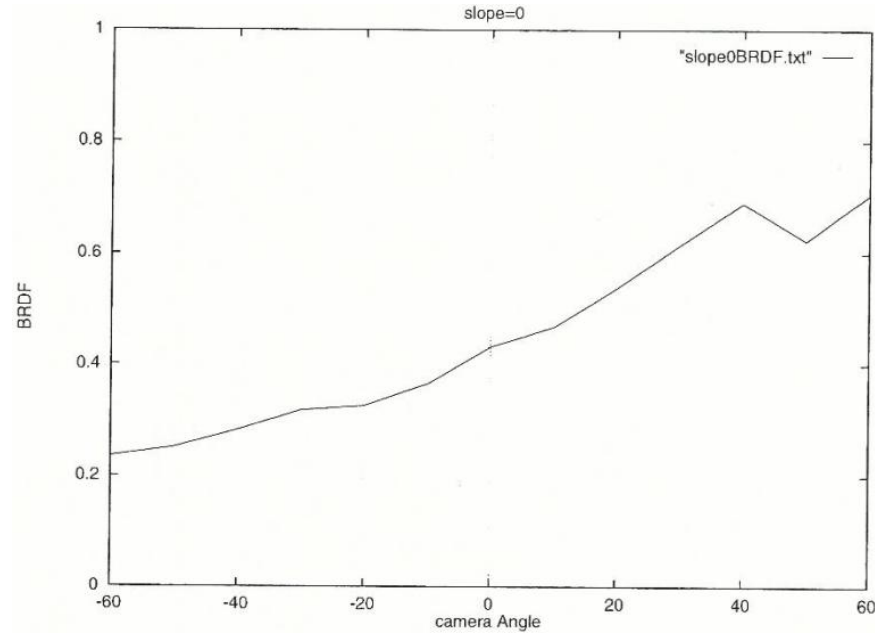

Fig.7. BRDF of the slope zero surface based on proposed surface model derived from the MCRT simulation

TABLE I. Given PARAmetrs For thye Proposed SURface Model

(a)Geometric relations among the sun, sensor and ground target

\begin{tabular}{|c|c|c|c|}
\hline$\theta_{s}$ & 41.0 & $\mathrm{~h}$ & 200 \\
\hline$\theta_{v}$ & $-60^{\circ} \sim 60^{\circ}$ & $\epsilon$ & $0^{\circ}$ \\
\hline$\Delta_{v}$ & 10 & $\Delta_{\epsilon}$ & $0^{\circ}$ \\
\hline$\alpha$ & 4 & & \\
\hline
\end{tabular}

(b)Parameters for ellipsoid

\begin{tabular}{|c|c|c|}
\hline & left & right \\
\hline $\mathrm{a}$ & 2.5 & 2.0 \\
\hline $\mathrm{b}$ & 5.0 & 4.0 \\
\hline $\mathrm{d}$ & 5.4 & 4.2 \\
\hline $\mathrm{t}$ & 5.0 & 4.0 \\
\hline
\end{tabular}

There is the hot spot at around 40 degree of observation angle (camera angle) because of solar zenith angle is 41 degree.

\section{B. Comparison between Linear and Nonlinear Mixture Model of the Mixed Pixels in Concern}

Figure 8 (a) shows how the surface of nonlinear mixture model looks like while Figure 8 (b) and (c) shows, respectively, how the surface of linear mixture model looks like (Top view).

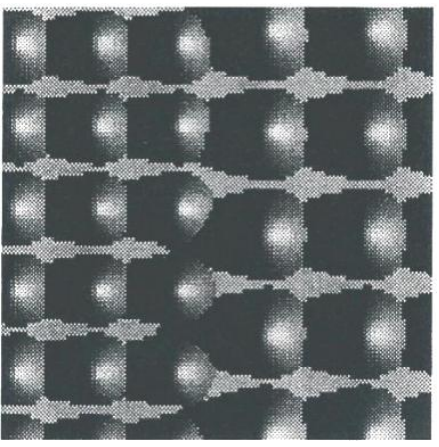

(a)Nonlinear

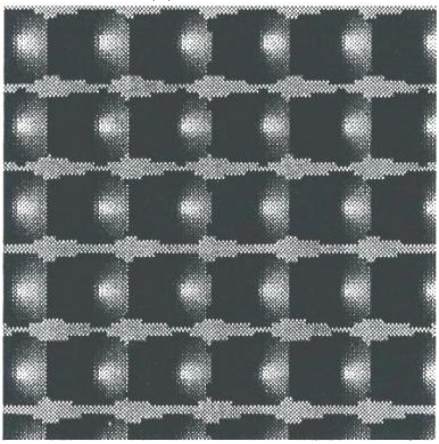

(b)Linear (right)

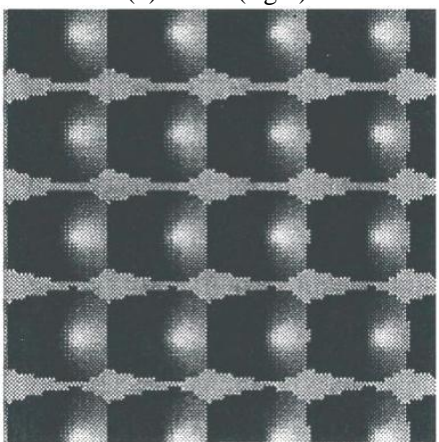

(c)Linear (left)

Fig.8. Examples of outlooks of the surface with ellipsoidal shapes of elements based surfaces for linear and nonlinear mixed pixel models.

Nonlinear mixed pixel model assumes the flat surface of Figure 8 (a) of pixel with half mixing ratios for each surface. Then, MCRT simulation is conducted once. On the other hand, linear mixed pixel model assumes the flat surface of the linear right (Figure 8 (b)) and the linear left Figure 8 (c)), separately. Then, MCRT simulation result for each surface is combined together after MCRT simulation.

BRDF derived from the MCRT simulation with the linear and nonlinear surface mixture models is shown in Figure 9. 
The parameters for this simulation are shown in Table 2. Hot spot is situated at around observation angle of 40 degree. The difference between linear and nonlinear models is quite small.

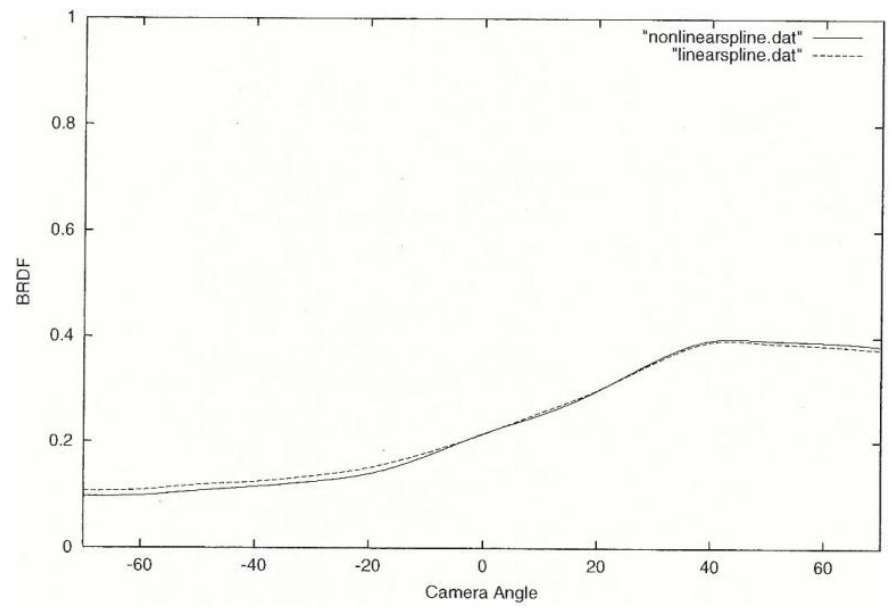

Fig.9. BRDF derived from the MCRT simulation with the linear and nonlinear surface mixture models

TABLE II. Given PARAMETRS For THYe Proposed SuRface Model

(a)Geometric relations among the sun, sensor and ground target

\begin{tabular}{|c|c|c|c|}
\hline$\theta_{s}$ & 36.9 & $\mathrm{~h}$ & 200 \\
\hline$\theta_{v}$ & $-70^{\circ} \sim 70^{\circ}$ & $\epsilon$ & $0^{\circ}$ \\
\hline$\Delta_{v}$ & 10 & $\Delta_{\epsilon}$ & $0^{\circ}$ \\
\hline$\alpha$ & 4 & & \\
\hline \multicolumn{4}{|c|}{ (b)Parameters for ellipsoid }
\end{tabular}

\begin{tabular}{|c|c|c|}
\hline & left & right \\
\hline $\mathrm{a}$ & 1.4 & 1.9 \\
\hline $\mathrm{b}$ & 8.5 & 11.5 \\
\hline $\mathrm{d}$ & 2.6 & 3.2 \\
\hline $\mathrm{t}$ & 4.1 & 4.5 \\
\hline
\end{tabular}

Dotted line in Figure 9 shows linear model derived BRDF while solid line shows nonlinear model derived BRDF. Linear model derived BRDF is little bit greater than that of nonlinear model derived BRDF. Due to multiple reflection between two slopes, BRDF decreases for the nonlinear mixture surface model.

\section{TOA Radiance Comparison between Linear and Nonlinear Mixture Models}

Top of the Atmosphere: TOA radiance $\left(\mathrm{W} / \mathrm{cm}^{2} / \mathrm{str}\right)$ is estimated based on MCRT simulation with the parameters of Table 2 as functions of optical depth of atmospheric molecule and aerosol. The MCRT simulation is conducted with the parameters shown in Table 2 except observation angle. The designated observation angle is zero of zenith angle, nadir view. Figure 10 (a) and (b) shows the difference between linear and nonlinear mixture surface models derived TOA radiances for the surface reflectance of 0.2 (a) and 0.4 (b), respectively. The vertical axis of Figure 10 shows nonlinear model based TOA radiance minus linear model based TOA radiance. TOA radiance depends on the reflectance significantly. These results are compared to those derived from MODTRAN 4. Both TOA radiances show good coincidence. Therefore, the proposed simulation model is validated.
The parameters for surface model of ellipsoidal shapes are changed. The TOA radiance with surface reflectance of 0.4 differences between linear and nonlinear models is shown in Figure 11 for aerosol optical depth of 0.1 and molecule optical depth of 0.1 . Figure 11 (a) shows the case that the parameter "a" is changed for left slope with keeping the parameter "a" for right slope at 1.9. The parameters for Figure 11 (b) to (i) are shown below,

(b)Left $\mathrm{a}=1.4$, right $\mathrm{a}=1.1-2.1$

(c)Left $b=10.9-11.5$, right $b=11.5$

(d)Left $b=8.5$, right $b=7.9-8.9$

(e)Left $\mathrm{d}=2.2-3.2$, right $\mathrm{d}=3.2$

(f)Left $\mathrm{d}=2.6$, right $\mathrm{d}=2.8-3.6$

(g)left $\mathrm{t}=3.7-4.5$, right $\mathrm{t}=4.5$

(h)Left $\mathrm{t}=4.1$, right $\mathrm{t}=4.1-4.9$

(i) $\varepsilon=0$-20 degree, $\mathrm{t}=0.0, \mathrm{n} 0.5,1.0$, aerosol optical depth $=0.2$, and molecule optical depth $=0.2$ where $\varepsilon$ denotes slope angle of the surface in concern.

The vertical axis of Figure 11 shows nonlinear mixture model based TOA radiance minus linear mixture model based TOA radiance.

In comparison to linear and nonlinear mixture models derived TOA radiance as a function of $b$ is not so significant comparing to those for the parameter a. On the other hand, the parameters of $\mathrm{d}$ and $\mathrm{t}$ are significant. Meanwhile, surface slope is much significant in particular for the slope angle is much larger than 15 degree.
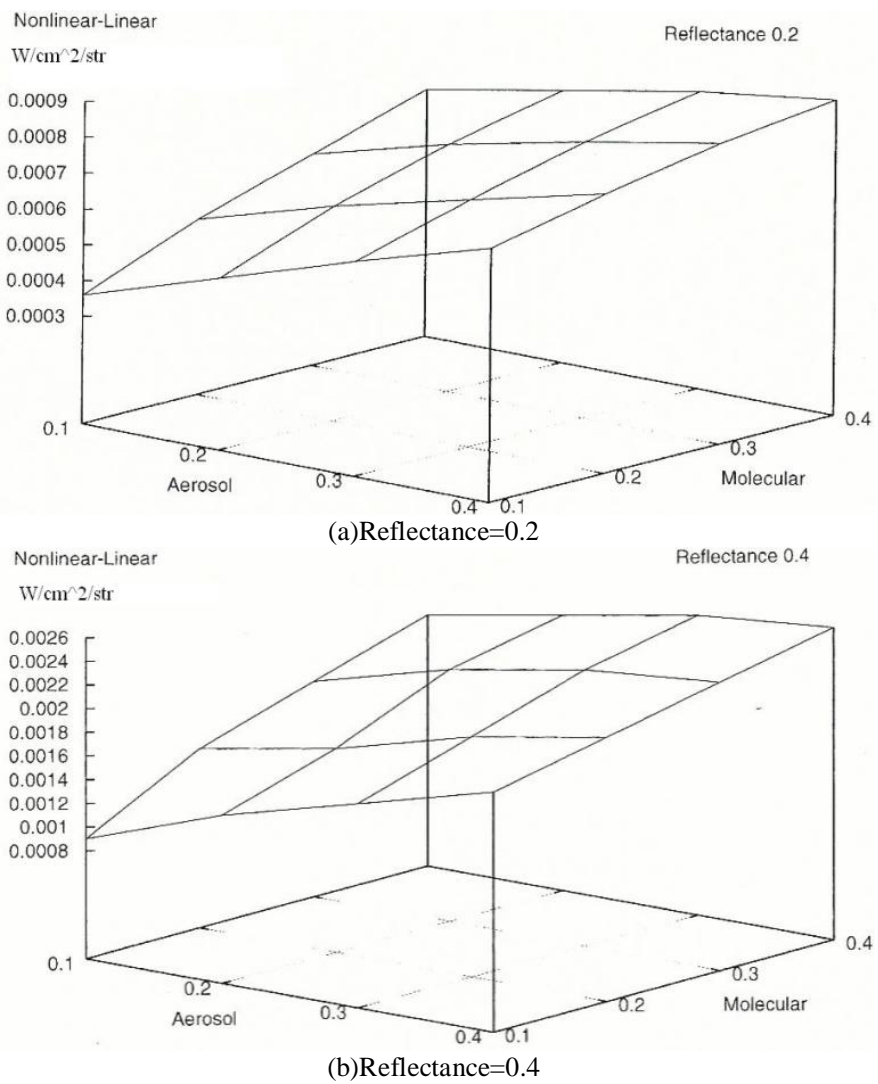

Fig.10. Estimated TOA radiances as function of aerosol and molecule optical depth as well as surface reflectance. 
(IJARAI) International Journal of Advanced Research in Artificial Intelligence,

Vol. 2, No.4, 2013
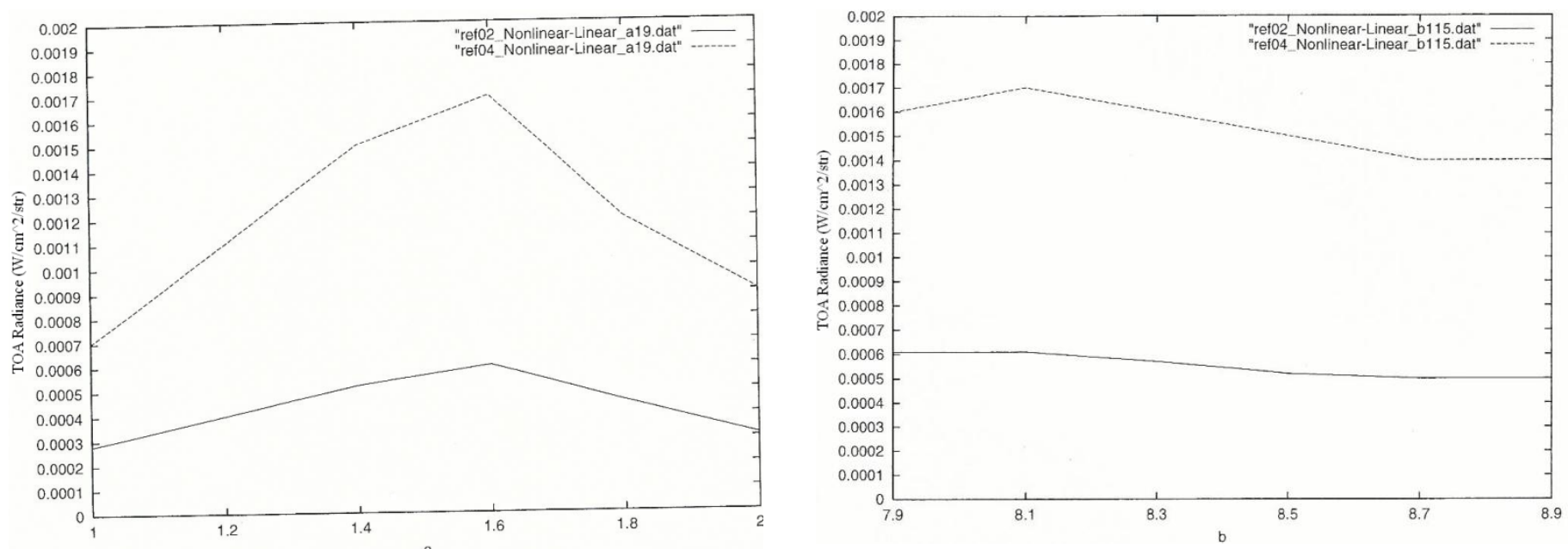

(a)Left $\mathrm{a}=1-2$, and right $\mathrm{a}=1.9$
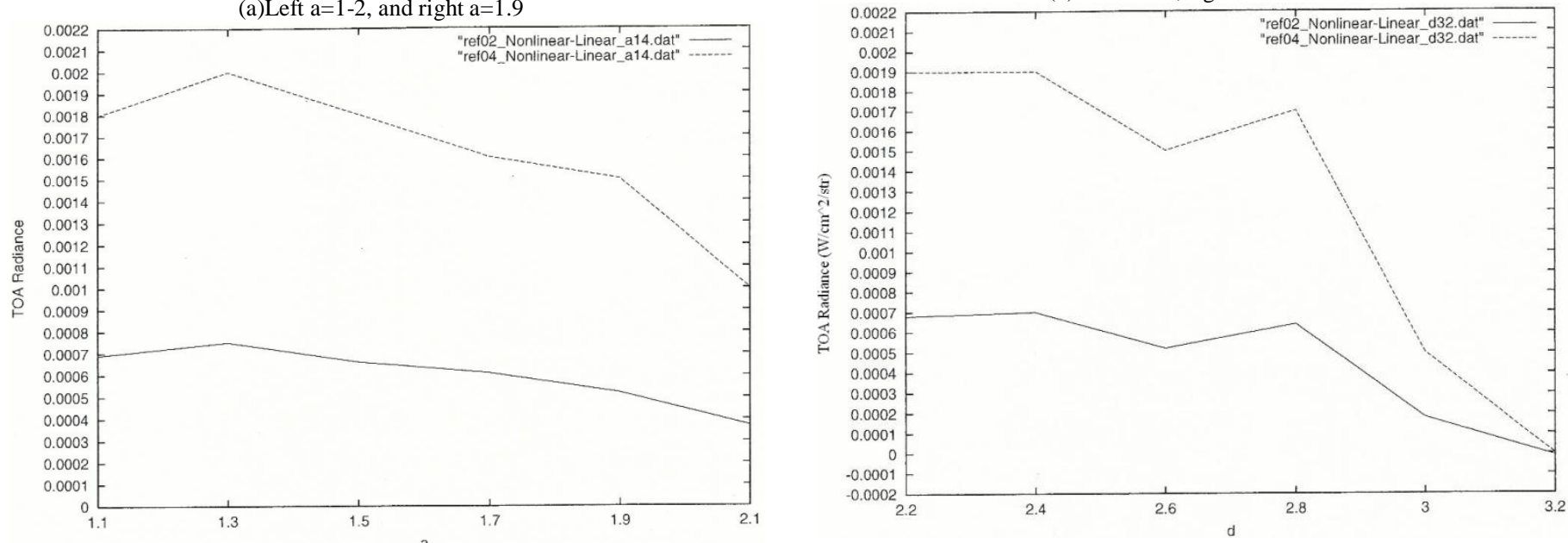

(b)Left a $=1.4$, right $\mathrm{a}=1.1-2.1$

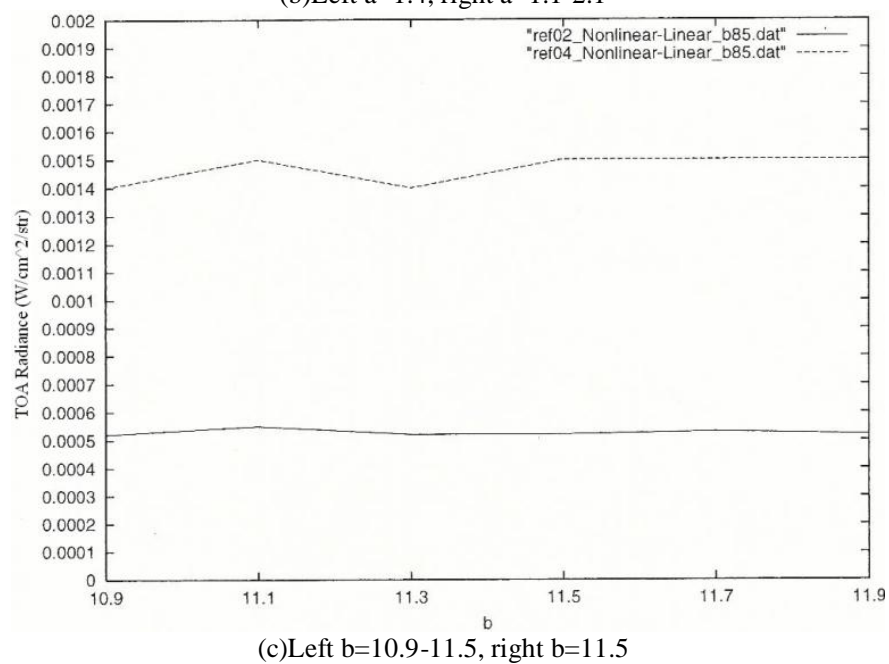

(e)Left $d=2.2-3.2$, right $d=3.2$

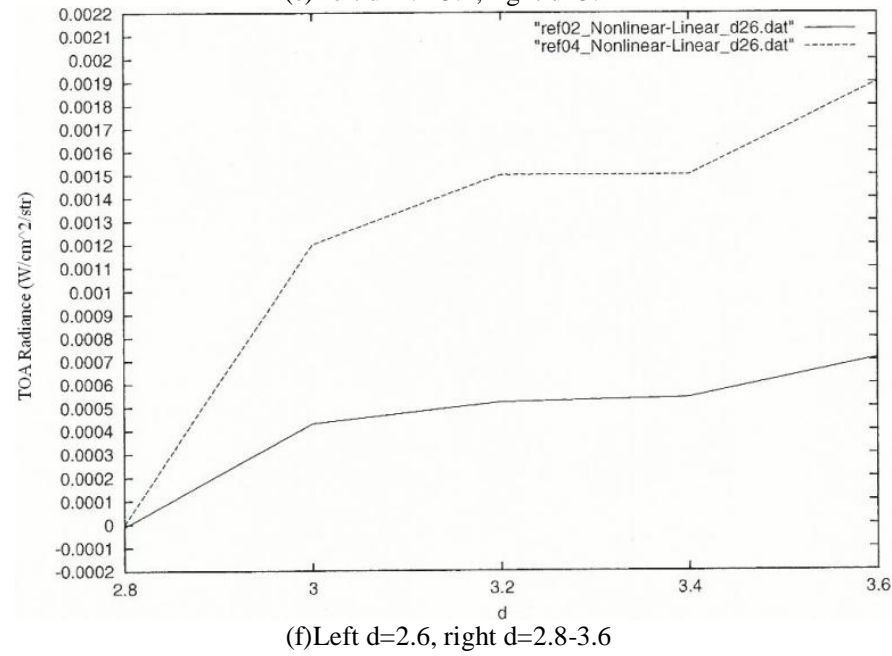



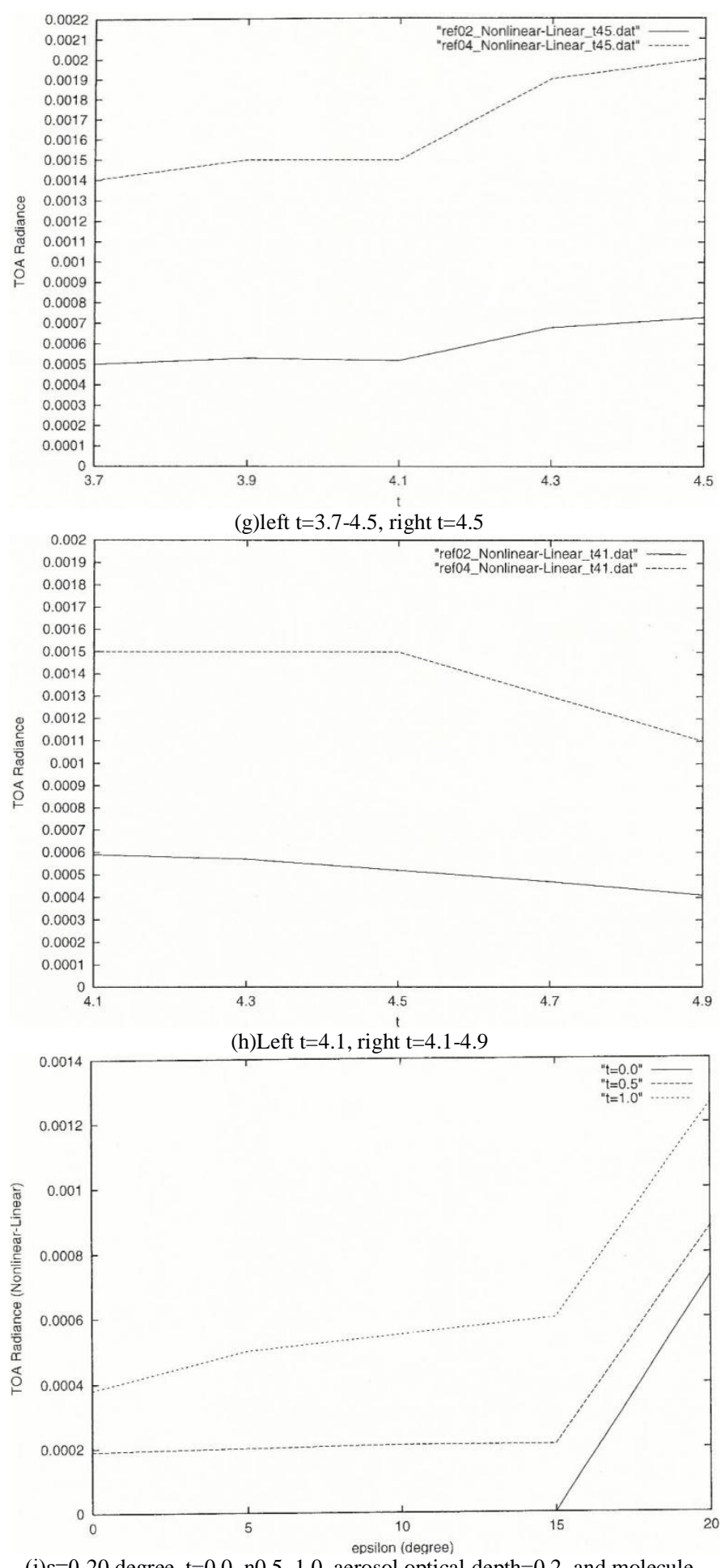

(i) $\varepsilon=0-20$ degree, $\mathrm{t}=0.0, \mathrm{n} 0.5,1.0$, aerosol optical depth $=0.2$, and molecule optical depth $=0.2$

Fig.11. TOA radiance difference between linear and nonlinear mixture models

\section{CONCLUSION}

Comparative study on linear and nonlinear mixed pixel models of which pixels in remote sensing satellite images is composed with plural ground cover materials mixed together, is conducted for remote sensing satellite image analysis. The mixed pixel models are based on Cierniewski of ground surface reflectance model.

The comparative study is conducted by using of Monte Carlo Ray Tracing: MCRT simulations. Through simulation study, the difference between linear and nonlinear mixed pixel models is clarified. Also it is found that the simulation model is validated.

In comparison to linear and nonlinear mixture models derived TOA radiance as a function of $b$ is not so significant comparing to those for the parameter a. On the other hand, the parameters of $d$ and $t$ are significant. Meanwhile, surface slope is much significant in particular for the slope angle is much larger than 15 degree.

\section{ACKNOWLEDGMENT}

The author would like to thank Mis. Reiko Inagaki for her efforts through experiments and simulations.

\section{REFERENCES}

[1] R.Singer and T.B.McCord, Mars; Large scale mixing of bright and dark surface materials and implications for analysis of spectral reflectance, Proc., 10th Lunar and Planetary Sci., Conf., 1835-1848, 1979.

[2] B.Hapke, Bidirection reflectance spectroscopy, I. Theory, Journal of Geophysical Research, 86, 3039-3054, 1981.

[3] R.N.Clark and T.I.Roush, Reflectance spectroscopy: Quantitative analysis techniques for remote sensing applications, Journal of Geophysical Research, 89, B7, 6329-6340, 1984.

[4] R.Singer, Near infrared spectral reflectance of mineral mixtures: Systematic combinations of pyroxenes olivine and iron oxides, Journal of Geophysical Research, 86, 7967-7982, 1974.

[5] B.Nash and J.Conel, Spectral reflectance systematic for mixtures of powered hypersthenes, labradoride and ilmenite, Journal of Geophysical Research, 79, 1615-1621, 1974.

[6] C.C.Borel and S.A.Gerst, Nonlinear spectral mixing models for vegetative and soils surface, Remote Sensing of the Environment, 47, 2, 403-416, 1994.

[7] J.Cierniewski, Geometrical modelling of soil bi-directional reflectance in the optical domain, Bougucki Scientific Publishers, 1999.

[8] K.Arai, Fundamental Theory on Remote Sensing, Gakujutu-toshoShuppan, Publishing Co. Ltd., 2001.

[9] Arai, K, Lecture Notes on Remote Sensing, Morikita-Shuppan, Co.Ltd., 2005

\section{AUTHORS PROFILE}

Kohei Arai, He received BS, MS and PhD degrees in 1972, 1974 and 1982, respectively. He was with The Institute for Industrial Science and Technology of the University of Tokyo from April 1974 to December 1978 also was with National Space Development Agency of Japan from January, 1979 to March, 1990. During from 1985 to 1987, he was with Canada Centre for Remote Sensing as a Post Doctoral Fellow of National Science and Engineering Research Council of Canada. He moved to Saga University as a Professor in Department of Information Science on April 1990. He was a councilor for the Aeronautics and Space related to the Technology Committee of the Ministry of Science and Technology during from 1998 to 2000 . He was a councilor of Saga University for 2002 and 2003. He also was an executive councilor for the Remote Sensing Society of Japan for 2003 to 2005 . He is an Adjunct Professor of University of Arizona, USA since 1998. He also is Vice Chairman of the Commission "A" of ICSU/COSPAR since 2008. He wrote 30 books and published 322 journal papers 